\title{
MELHORIA DO PROCESSO DE CORREÇÃO GEOMÉTRICA DE IMAGENS CBERS-CCD PELO USO DE AMOSTRAS GEORREFERENCIADAS CLASSIFICADAS
}

\section{Improvements over the geometric correction of CBERS-CCD images by using classified georeferenced samples}

\author{
Emiliano Ferreira Castejon ${ }^{1}$ \\ Leila Maria Garcia Fonseca ${ }^{2}$ \\ Carlos Henrique Quartucci Forster ${ }^{3}$ \\ 1,2 \{leila,castejon\}@dpi.inpe.br - Instituto Nacional de Pesquisas Espaciais - INPE - São José dos \\ Campos - São Paulo - Brasil \\ ${ }^{3}$ forster@ita.br - Instituto Tecnológico da Aeronáutica - ITA - São José dos Campos - São Paulo - Brasil
}

\section{Resumo:}

As imagens da série de satélites CBERS são distribuídas gratuitamente, mas para que seja possível utilizá-las, é necessário aplicar um método de correção geométrica. É proposta uma melhoria do processo de correção automática de forma a selecionar as melhores amostras de referência a partir das quais é possível definir pontos de controle usados para o cálculo dos parâmetros do modelo usado na correção. Para demonstrar a eficácia, o método proposto é aplicado em um conjunto de imagens CBERS usando as amostras de imagens selecionadas.

Palavras-chave: otimização, seleção, amostras, registro, imagens, sensoriamento, remoto.

\begin{abstract}
:
The CBERS satellite images are freely distributed, but a geometric correction method is required to allow their use. We propose improvements over the automatic correction method by selecting the best reference samples from which control-points are generated. Further, those tie points will be used to define the geometric model used on the correction process. To demonstrate the effectiveness and performance gain we applied the correction method to a set of selected CBERS images using the automatically selected samples.
\end{abstract}

Keywords: optimization, samples, selection, image, register, remote, sensing 


\section{Introdução}

O programa CBERS é o resultado de uma cooperação entre Brasil e China cujo objetivo é o desenvolvimento de uma série de satélites denominada CBERS (sigla para China-Brazil Earth Resources Satellite; em Português, Satélite Sino-Brasileiro de Recursos Terrestres). Três satélites desta série já foram lançados: CBERS-1, 2 e 2B. Um dos sensores a bordo do CBERS-2B é a câmera CCD cujas imagens adquiridas são distribuídas gratuitamente no catálogo online de imagens CBERS. Tais imagens já possuem um certo nível de correção, mas ainda existem erros de geometria e posicionamento que devem ser corrigidos ou pelo menos reduzidos. Cabe ao usuário efetuar a correção usando o processo conhecido como registro de imagens (Fonseca 1999), que está entre as etapas iniciais de processamento de imagens. Este processo demanda tempo e atenção do usuário para que uma imagem corrigida de boa qualidade seja obtida.

A correção geométrica de imagens envolve a remoção de distorções geométricas inseridas durante o processo de imageamento, fazendo com que não haja correspondência entre as posições dos elementos de imagem e as posições dos objetos na superfície da Terra. Dentre as formas automáticas de correção, está aquela onde é feito um refinamento da correção de posicionamento do satélite, que envolve muitas vezes apenas um deslocamento. Para tal um conjunto de pequenos recortes de imagens georreferenciadas, chamados kernels, é usado como dado de referência. Os kernels podem ser gerados a partir de imagens de outros satélites, como os da série LANDSAT, ou até mesmo imagens da própria série CBERS, que já tenham a geometria corrigida.

O objetivo deste trabalho é apresentar um método para selecionar o melhor conjunto de amostras de imagens que será usado no processo automático de correção geométrica de forma a reduzir o tempo de computação, dado que um conjunto menor de amostras será processado. Na seção seguinte são descritas as características das imagens CBERS/CCD e os métodos tradicionalmente usados para corrigir imagens de outros sensores semelhantes. Na seção 3, são apresentadas etapas do método proposto, que vão desde a geração das imagens de referência até a geração de amostras usadas para o treinamento de classificadores destas mesmas imagens. $\mathrm{Na}$ seção 4 são avaliados diferentes modelos de classificadores treinados usando as amostras citadas anteriormente. Na seção 5 avalia-se o desempenho computacional obtido no processo de correção das imagens CBERS usando apenas aqueles kernels selecionados automaticamente pelos classificadores. Será demonstrado que é possível aumentar sensivelmente o número de kernels que efetivamente contribuem no processo de correção e também que é possível reduzir razoavelmente o tempo de processamento pela aplicação de um processo de classificação simples.

\section{Correção de imagens CBERS}

A questão do nível de qualidade de uma imagem está diretamente relacionada ao processo de aquisição e à influência de fatores que podem inserir distorções geométricas nos produtos gerados. Um exemplo semelhante às distorções presentes nas imagens da série CBERS2 (D' Alge, Souza, and Erthal 2004), são as distorções presentes em dados da série de satélites de 
média resolução LANDSAT, que são causados por fatores internos inerentes à sua construção e fatores externos.

Para reduzir estes e outros tipos de distorções introduzidas na formação da imagem é aplicado um modelo fotogramétrico baseado no princípio das equações de colinearidade (Gugan and Dowman 1988)(Kim and Dowman 2006). Estas equações relacionam coordenadas da imagem (linhas e colunas) com coordenadas 3D em um sistema de referência do espaço objeto (latitude, longitude e altitude).

\subsection{Características das Imagens CBERS/CCD}

O deslocamento na órbita do satélite fora da faixa permitida provoca um erro na posição da cena de forma que os objetos aparecem deslocados em imagens adquiridas da mesma cena. Este problema existe nas imagens CBERS-CCD, e se deve a falhas nos dados usados para atualizar o posicionamento do satélite em sua órbita de forma que esta possa ser modificada caso necessário (Silva 2007) (D’ Alge, Souza, and Erthal 2004).

Para prover dados adequados para os usuários de sensoriamento remoto, diferentes níveis de correção das imagens CBERS são realizados: Nível 0 (imagens em estado bruto para armazenamento); Nível 1 (possuem apenas ajustes na radiometria como remoção de ruído, calibração, etc.); Nível 2 (possuem ajustes na radiometria e ajustes na geometria baseados em parâmetros do satélite para modelar e corrigir as distorções inseridas pelo sensor e fatores externos. Devido a erros de posicionamento do satélite, distorções geométricas ainda podem existir neste nível de correção); Nível 3 (melhoria na geometria do nível 2 pelo uso de pontos de controle obtidos a partir de dados de referência que possuem coordenadas geodésicas corretas e conhecidas); Nível 4 (também conhecido como ortoretificação, onde dados referentes ao relevo do terreno imageado são levados em consideração no processo de correção).

\subsection{O Uso de Imagens de Referência}

Para que os erros de posicionamento das imagens do nível 2 sejam corrigidos, vários métodos foram propostos (Marcato Junior and Tommaselli 2013) (Fedorov 2002) (Fonseca,1999). Estes métodos consistem em localizar pontos homólogos (correspondentes) na imagem que se deseja corrigir e no dado de referência. Devido às características das imagens, estes métodos não possuem a robustez necessária para que seja sempre possível efetuar a correção e assim, os usuários precisam recorrer ao método de registro manual (Zitová and Flusser 2003).

Pesquisadores da USGS (United States Global Land Survey) propõe um método para gerar imagens de satélites da série LANDSAT com correção de nível 3 (USGS Publications Warehouse 2006). O método baseia-se na utilização de uma base de dados de referência, da qual pontos de controle são extraídos. A base de dados de referência foi criada como resultado de colaboração entre a agência espacial NASA e o instituto USGS com o objetivo de criar três

Bol. Ciênc. Geod., sec. Artigos, Curitiba, v. 21, no 4, p.658-673, out-dez, 2015. 
conjuntos globais de imagens corrigidas da série de satélites LANDSAT entre os anos de 1970, 1990 e 2000 cuja geometria e posicionamento espacial são verificados e garantidos.

Por questões de otimização e capacidade de armazenamento, apenas partes desta base de dados são efetivamente armazenadas e utilizadas. Pequenos recortes, (com tamanho aproximado de 129x129 pixels) denominados kernels ou chips, são gerados a partir das imagens processadas e armazenados.

\subsection{O Processo de Correção}

De forma semelhante ao que é feito para as imagens LANDSAT, o processo de correção geométrica de uma imagem CBERS se inicia na etapa de localização de pontos homólogos entre a imagem de ajuste e os kernels compatíveis (Castejon 2011). Para se definir qual é posição geográfica correta de todos os pontos da imagem de ajuste (imagem com nível 2 de correção, que deve ser corrigida) é necessário usar um modelo matemático que associe posições de pontos da imagem nas respectivas coordenadas geográficas. Os parâmetros do modelo podem ser definidos pelo uso de pontos de controle, cujas coordenadas são definidas quando existe correspondência de algum kernel com a respectiva posição na imagem de ajuste.

Para cada imagem submetida ao processo de correção são usados kernels da base de referência que tenham alguma intersecção com a região geográfica da imagem a ser corrigida. A correta posição do kernel sobre a imagem é definida dentro de uma janela de busca, que maximiza o valor de alguma métrica de similaridade. As dimensões da janela de busca são definidas tomando-se como base o conhecimento a priori do erro médio das imagens do sensor em questão. Para o caso da correção das imagens CBERS-CCD a janela de busca pode ter até $500 \times 500$ pixels, o que corresponde a um erro de $10 \mathrm{Km}$. Neste caso a medida de similaridade usada é a medida de correlação (Pratt 1974), dado que a correlação tem boa relação eficácia/custo computacional quando comparada a outras métricas (Castejon et al. 2009).

Devido a diversos fatores, como a baixa qualidade de alguns kernels, oclusão de objetos, presença de artefatos na imagem (ruído, dados faltantes ou pontos com valores que não representam corretamente os objetos imageados) ou áreas homogêneas na imagem, pode ocorrer o casamento incorreto de alguns deles com as respectivas posições na imagem. Nestes casos seriam gerados pontos de controle incorretos que invalidariam o processo de correção. Para resolver este problema é usado o método RANSAC (Fischler and Bolles 1981), que iterativamente refina os parâmetros de um modelo de transformação geométrica pré-definido e remove os pontos de controle incorretos. Tendo-se os parâmetros do melhor modelo de transformação é possível gerar a imagem corrigida.

A cada execução do processo de correção de uma imagem são usados vários kernels selecionados da base de referência. Ao final do processo nem todos eles fazem parte do grupo que efetivamente define o modelo da transformação, que modela a distorção existente na imagem. E assim eles podem ser agrupados da seguinte forma: O primeiro grupo é daqueles descartados - kernels cujo maior valor do coeficiente de correlação encontrado no processo de correlação está abaixo de um limiar pré-definido e, desta forma, não são utilizados. O segundo grupo é daqueles que foram filtrados - o kernel foi incorretamente localizado sobre a imagem a ser ajustada e a relação geométrica deste kernel com os demais não gera uma transformação geométrica aceitável. O terceiro grupo são os kernels que foram localizados com sucesso e têm 
consistência geométrica entre si. Este último grupo é efetivamente usado para gerar a imagem corrigida.

\section{Metodologia}

Neste tópico são apresentados os passos e técnicas usados para atingir o objetivo proposto. Inicialmente é feita a seleção de quais imagens referência LANDSAT serão usadas como referência para corrigir imagens CBERS. Da correção de todas as imagens serão gerados dados estatísticos sobre o uso dos kernels durante o processo. Com esta informação será feita uma análise para definir o melhor modelo de classificação de kernels, com o qual será possível avaliar o ganho obtido na correção geométrica pela aplicação do método.

\subsection{Seleção das Imagens}

A estratégia seguida neste trabalho foi a de utilizar imagens CBERS-CCD com nível 2 de correção, adquiridas a partir do catálogo de imagens do Instituto Nacional de Pesquisas Espaciais - INPE (://www.dgi.inpe.br/CDSR/), tomando como referência a grade de órbitas CBERS. O primeiro critério de seleção utilizado foi o número de cenas (nível 2 de processamento) disponíveis por órbita/ponto. O uso daquelas regiões com maior quantidade de cenas possibilita a geração de maior quantidade de dados estatísticos de utilização de kernels, calculados quando cada região selecionada é submetida ao processo de correção para a geração das respectivas imagens corrigidas (nivel 3 de processamento).

O segundo critério de seleção foi localizar, dentre as órbitas com maior disponibilidade de imagens, aquelas com menor cobertura de nuvens. A cobertura de nuvens dificulta a correlação de kernels pela oclusão de alvos na cena e pode gerar dados estatísticos incorretos.

Para cada região foram selecionadas várias cenas CBERS2/CBERS2B CCD (banda 4). As respectivas cenas LANDSAT5-TM e LANDSAT7-ETM (com nível de correção 3) que possuam alguma sobreposição geográfica também foram selecionadas a partir da base de imagens de referência USGS. Foram usadas imagens de datas diferentes e, sempre que possível, a escolha das datas foi feita de forma a melhor distribuir as aquisições durante as quatro estações anuais entre os anos de 2004 a 2009. Isto é feito para que dados de uso fossem gerados englobando situações onde existem, por exemplo, diferenças de iluminação e diferenças temporais. As imagens selecionadas são: 22 cenas da órbita 153/114, localizada na região nordeste do Brasil e cobre o município de Paratinga; 22 cenas da órbita 153/115 que está localizada na região nordeste do Brasil e cobre o município de Bom Jesus da Lapa; 23 cenas da órbita 162/126, localizada na região centro-oeste do Brasil e que cobre o município de Iguatemí; 18 cenas da órbita 162/126 que está localizada na região sudeste do Brasil e cobre o município de Pitanguí. A Figura Figura mostra uma cena para cada uma das órbitas usadas. 


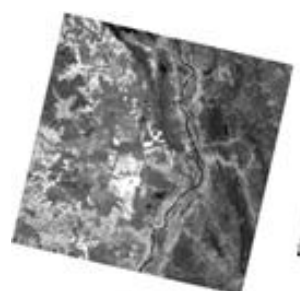

A

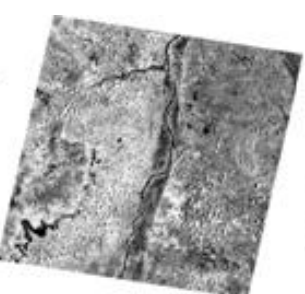

B

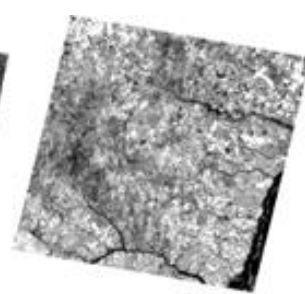

C

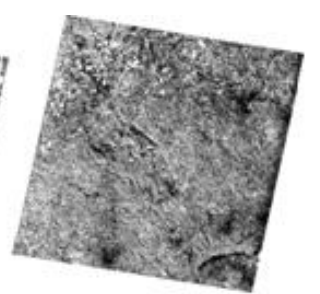

$\mathrm{D}$

Figura 1: Órbitas escolhidas ( A- CBERS2B - órbita 153/114 - banda 4- Paratinga, BCBERS2B - órbita 153/115 - Banda 4 - Bom Jesus da Lapa, C- CBERS2 - órbita 162/126 banda 4 - Iguatemi, D- CBERS2 - órbita 153/122 - banda 4 - Pitanguí

\subsection{Geração de Kernels Candidatos}

Nesta etapa são gerados kernels a partir das imagens de referência selecionadas. Cada kernel é uma sub-imagem LANDSAT cuja coordenada central é definida pelo uso do operador de Moravec (Moravec 1977). Este operador funciona de forma semelhante a um filtro linear, onde os pixels com maior resposta máxima local à aplicação do operador representam posições onde existe maior variação de sinal na imagem (bordas e cantos de objetos, por exemplo). As áreas da imagem que contém maiores valores de resposta do operador definem as posições para o recorte de kernels (Castejon 2011).

O operador foi implementado e executado em um software baseado na biblioteca de software TerraLib (Câmara et al. 2008) de modo que ao final do processo foram gerados 2527 kernels candidatos (para todas as imagens de referência) com dimensões de 129 por 129 pixels.

\subsection{O Uso de Atributos Texturais de Kernels}

Ao final da execução de um processo de correção tem-se a informação do estado final da utilização de cada kernel, entretanto somente esta informação não é suficiente para treinar um classificador. Atributos adicionais podem ser calculados e seus valores agregados às estatísticas para descrever e representar cada kernel. Atributos simples podem ser extraídos com base nos valores de estatísticas de primeira ordem (entropia, desvio padrão, variância, moda, média, etc.).

Trabalhos na área de classificação de imagens baseada em objetos (Herold, Liu, and Clarke 2003) têm demonstrado a eficácia da utilização de atributos texturais para a identificação de classes de cobertura do solo intraurbano em imagens de alta resolução espacial. Algumas das métricas mais usadas são aquelas derivadas de matrizes de co-ocorrência de níveis de cinza (Haralick 1979). Estas medidas agregam informação da relação espacial entre cada elemento de 
imagem e sua vizinhança (sob um raio e uma direção pré-definidos) com a criação da matriz de co-ocorrências. Desta forma, em analogia aos processos estatísticos de primeira ordem (como média, variância, e outros) podem ser calculados os respectivos atributos estatísticos texturais de segunda ordem.

Os atributos utilizados neste trabalho são aqueles calculados em função da matriz de coocorrências de Haralick, tomadas duas direções para o cálculo: leste (0 graus) e norte ( 90 graus). Os atributos são: Dissimilaridade, contraste, entropia, homogeneidade, correlação, média, desvio padrão, energia e Chi-square.

\subsection{O Uso do Atributo de Registrabilidade}

Imagens com feições expressivas e maior quantidade de informação são mais sensíveis a deformações geométricas. Baseado nesta afirmativa, Chalermwat (Chalermwat 1999) propôs uma medida de registrabilidade que expressa a probabilidade de se obter um bom registro de imagem. Imagens com feições pouco definidas ou com pouca quantidade de informações (homogêneas) tendem a gerar baixos valores de registrabilidade, ao contrário das imagens com feições bem definidas, que obtém valores mais elevados de registrabilidade.

O tipo de transformação que modela a distorção geométrica é um dos parâmetros usados no cálculo do índice de registrabilidade. Chalermwat usa a transformação de rotação. Neste caso, várias versões rotacionadas da imagem são geradas. Cada uma delas é comparada com a imagem original segundo a métrica de correlação cruzada normalizada, dada pela equação 1 . O valor do índice da registrabilidade é calculado como sendo o desvio padrão dos valores de correlação obtidos para todas as comparações como mostrado na equação 2 .

$$
C(i, j)=\frac{\sum_{l=0}^{k-1} \sum_{m=0}^{L-1}\left(W_{Z}(l, m)-\mu_{W}\right)\left(S_{i, j}(l, m)-\mu_{S}\right)}{\sqrt{\sum_{l=0}^{k-1} \sum_{m=0}^{L-1}\left(W_{Z}(l, m)-\mu_{W}\right)^{2} \sum_{l=0}^{k-1} \sum_{m=0}^{L-1}\left(S_{i, j}(l, m)-\mu_{S}\right)^{2}}}
$$

Onde:

$W_{Z}$ é o valor do nível de cinza de um elemento na imagem original.

$S_{i, j}$ é o valor do nível de cinza de um elemento do kernel transformado.

$\mu_{W}$ é a média dos valores de nível de cinza dos pontos dentro da região considerada na imagem original.

$\mu_{S}$ é a média dos valores de nível de cinza dos pontos do kernel transformado.

$$
R=\sqrt{\frac{\sum^{n}\left(C_{n}-\mu_{C}\right)^{2}}{n}}
$$

Onde:

n é o número de transformações aplicadas sobre o kernel.

Bol. Ciênc. Geod., sec. Artigos, Curitiba, v. 21, no 4, p.658-673, out-dez, 2015. 
$\mathrm{C}_{\mathrm{n}}$ são os valores de correlação referentes a cada transformação.

$\mu_{\mathrm{C}}$ é o valor médio de correlação.

Eikvil (Eikvil, Holden, and Huseby 2009) usa a medida de registrabilidade para identificar as melhores regiões em imagens para registro e, de forma semelhante, no trabalho aqui proposto, são utilizados dois valores de registrabilidade: um relacionado a uma transformação de rotação (em relação ao centro da imagem) e outro valor relacionado a uma transformação de translação.

Usando amostras de kernels de imagens LANDSAT foi verificado que existe uma tendência de que o valor da registrabilidade (para o caso onde a transformação usada é rotação) se estabilize em torno de 0,25 a partir de 12 repartições do ciclo trigonométrico (12 valores de ângulos diferentes com incrementos de 3,75 graus decimais). De forma análoga foi verificado que existe uma tendência de que o valor da registrabilidade (para o caso onde a transformação usada é de deslocamento) se estabilize em torno de 0,2 quando o valor do deslocamento máximo alcança o valor 8 (Castejon 2011).

O número de repartições do ciclo trigonométrico e o valor de deslocamento máximo para os casos onde o valor da registrabilidade se estabiliza são tomados como padrão para o cálculo do valor de registrabilidade para todos os kernels.

\subsection{Correção das Imagens}

Para gerar a informação estatística de qual é a participação de cada kernel no processo de correção geométrica todos eles são usados para corrigir todas as imagens CBERS selecionadas. São utilizados os seguintes parâmetros para a correção de todas as cenas: Dimensão da área de busca - fixado em 10000 metros de modo a cobrir os erros das cenas utilizadas. Valor mínimo de correlação - 0,2 (valor empírico de referência escolhido em função de testes executados nas imagens selecionadas). Valor máximo de erro - 20 metros (valor máximo de erro de posicionamento desejado, compatível a 1 ponto de imagem para imagens CBERS/CCD). Dado que modelos polinomiais exigem não apenas uma quantidade razoável de pontos de controle, mas também uma boa distribuição destes pontos ao longo de toda a imagem foi fixada uma porcentagem mínima de área coberta por pontos de controle de $30 \%$. O modelo de transformação usado foi o da afinidade, que é suficiente para corrigir as distorções presentes nas imagens CBERS/CCD selecionadas (Queiroz 2002).

A tabela 1 mostra o resultado da correção das imagens escolhidas usando todos os kernels (Castejon, Fonseca, and Arcanjo 2013). Por ela é possível perceber que houve uma grande quantidade de kernels descartados e incorretamente correlacionados. Isto contribui para o aumento no tempo de execução. As colunas representam respectivamente o número médio de kernels descartados (com nível de coeficiente máximo de correlação abaixo do limiar usado), o número médio de kernels correlacionados (com nível de coeficiente máximo acima do limiar usado), o número médio de kernels com boa geometria (aqueles que foram selecionados pelo método RANSAC modificado e que têm consistência geométrica e resíduo inferior ao valor de limiar de um ponto de imagem. Estes são os kernels que efetivamente são usados na geração da imagem corrigida), a porcentagem média da área coberta pelo fecho convexo gerado pelos centros dos kernels de boa geometria em relação a área total da imagem), o tempo médio gasto 
para efetuar a busca da melhor posição dos kernels e o estado final da correção, que é a taxa média de imagens corrigidas por

Tabela 1 - Resultado da correção geométrica órbita.

\begin{tabular}{c|c|c|c|c|c|c}
\hline Órbita & $\begin{array}{c}\text { Kernels } \\
\text { Descartados }\end{array}$ & $\begin{array}{c}\text { Kernels } \\
\text { Correlacionados }\end{array}$ & $\begin{array}{c}\text { Kernels com Boa } \\
\text { geometria }\end{array}$ & $\begin{array}{c}\text { Porcentagem da } \\
\text { Área coberta }\end{array}$ & $\begin{array}{c}\text { Tempo de } \\
\text { Execução (seg) }\end{array}$ & $\begin{array}{c}\text { Estado final Da } \\
\text { correção }\end{array}$ \\
\hline Paratinga & 80,09 & 80,29 & 25,43 & 21,85 & 3258,90 & $0,28(6 / 21)$ \\
\hline $\begin{array}{c}\text { Bom Jesus } \\
\text { da Lapa }\end{array}$ & 60,13 & 42,95 & 9,72 & 21,95 & 1790,95 & $0,31(7 / 22)$ \\
\hline Pitangui & 103,11 & 125,05 & 65,77 & 37,97 & 4597,55 & $0,77(14 / 18)$ \\
\hline Iguatemí & 48,79 & 54,75 & 2,62 & 6,62 & 1830,87 & $0,08(2 / 24)$ \\
\hline
\end{tabular}

Um dos parâmetros do processo de correção descrito é o valor máximo de erro por ponto de controle, o implica em que todos os pontos de controle gerados têm erro máximo de um pixel (para os casos onde houve sucesso na correção). Nos resultados obtidos, o erro médio quadrático tem valor em torno de 1,0 para todos os casos onde houve sucesso. Isto se deve principalmente ao método de seleção de pontos de controle usado (RANSAC), que sempre busca gerar o maior conjunto de pontos de controle geometricamente consistentes possível, obedecendo ao valor máximo de erro escolhido. Por consequência disto o valor médio de erro quadrático também tem valor em torno de 1,0 para todas as órbitas. A mesma situação ocorrerá na segunda rodada de correções. Assim, por questões de simplicidade esta informação não é apresentada na tabela Erro! Fonte de referência não encontrada.

Ao final do processo de correção geométrica é possível rotular cada kernel de acordo com o estado final de seu uso (se ele foi usado com sucesso, se foi descartado, etc.). Um mesmo kernel pode ser usado várias vezes, dado que várias imagens são corrigidas para cada cena (diferentes datas de aquisição). O mesmo ocorre para o caso da correção de cenas adjacentes, como as cenas de Paratinga e cenas de Bom Jesus da Lapa. Nesta situação pode ocorrer de um mesmo kernel ser utilizado na correção das duas ou mais imagens.

\section{6 Índice de Qualidade do Kernel}

Levando em consideração os rótulos que o estado final de uso de cada kernel pode receber ao final do processo de correção de uma imagem, é possível gerar um conjunto de estatísticas onde a cada kernel é associado um valor do índice de qualidade Q, definido na equação 3:

$$
Q=\frac{S}{D+F+S}
$$

Onde Q é o índice de qualidade do kernel, cuja faixa de valores está no intervalo [0,1]. D é o número ocorrências em que o kernel foi selecionado para ser usado na correção, mas durante o processo foi descartado. F é o número de ocorrências em que o kernel foi filtrado por inconsistência geométrica. S é o número de ocorrências em que o kernel foi usado com sucesso. 


\subsection{Geração de Amostras para Classificação}

Pela análise do índice de qualidade dos kernels foi verificado que a maioria deles possui baixo índice de qualidade. É desejado separá-los em duas classes: classe de kernels bons (aqueles que efetivamente são úteis na correção) a classe de kernels ruins (aqueles que não contribuem na correção e apenas aumentam o tempo computacional). A separação é feita segundo um valor de limiar de separação sobre o índice de qualidade de kernels, mostrado pela equação 3.

Dependendo do valor de limiar de separação escolhido poderá haver discrepância entre o número de amostras em cada classe, o que pode influenciar negativamente no treinamento de um classificador. A forma escolhida para determinar o melhor valor do limiar de separação foi o estudo do comportamento dos atributos segundo diferentes valores de limiar sobre o índice de qualidade dos kernels.

Foram gerados nove pares de conjuntos de amostras selecionadas a partir do conjunto de estatísticas de uso dos kernels original com valores de limiar de separação de qualidade variando de 0,1 a 0,9. Todos os conjuntos têm o mesmo número de amostras para as duas classes de kernels (bons e ruins). Para cada conjunto é definido um limiar de índice mínimo de qualidade e rotulam-se como amostras de bons kernels todas aquelas cujo valor índice excede o limiar. Além disto, também são agregados os atributos extraídos do kernel (estatísticos, texturais, etc.), citados anteriormente. Aleatoriamente é selecionada a mesma quantidade de kernels ruins (dentre aqueles cujo valor índice é inferior ao limiar). Para cada conjunto são geradas as respectivas amostras, como descrito na próxima seção.

\subsection{Avaliação de Atributos}

Dentre as razões para reduzir o número de atributos no processo de classificação está a redução da complexidade computacional. Usando apenas os melhores atributos é possível calcular os parâmetros de um classificador que seja capaz de predizer com uma taxa maior de acertos.

Da etapa anterior são gerados vários conjuntos de amostras de treinamento. O próximo passo é determinar quais são atributos que melhor separam as classes. Para tal, foi aplicada a técnica de seleção de atributos baseada no trabalho de Wei Deng (Deng et al. 2008) e L. Yu (Yu and Liu 2004), que usam a razão de Fisher (Fisher 1936) para ordenar os atributos em ordem decrescente. Os atributos com os maiores valores da razão de Fisher são selecionados até que o percentual de valores acumulados de Fisher ultrapasse 0,99. Atributos com valores de Fisher muito baixos são descartados.

A tabela

Tabela foi gerada usando os conjuntos de amostras definidos na etapa anterior. Nela estão listados atributos mais relevantes, separados por conjunto de amostras, segundo o limiar sobre o valor do índice de qualidade. Os atributos são apresentados em ordem decrescente de importância. Pelos resultados é possível verificar que os atributos mais importantes são entropia (expressa a quantidade de desordem na imagem), contraste (representa a quantidade de variações locais na imagem) e dissimilaridade (mede a relação de ocorrência de pares de valores de níveis 
de cinza na imagem), dado que são aqueles que aparecem com maior frequência, considerando todos os conjuntos de amostras.

Tabela 2 - Melhores atributos por conjunto de amostras

\begin{tabular}{|c|c|}
\hline $\begin{array}{l}\text { Valor do limiar sobre } \\
\text { o índice de qualidade }\end{array}$ & Melhores atributos \\
\hline 0,1 & $\begin{array}{l}\text { Dissimilaridade Haralick } 0^{\circ} \text {, Contraste Haralick } 0^{\circ} \text {, Dissimilaridade Haralick } 90^{\circ} \text {, Entropia Haralick } 0^{\circ} \text {, Entropia } \\
\text { Haralick } 90^{\circ} \text {, Homogeneidade Haralick } 0^{\circ} \text {, Homogeneidade Haralick } 90^{\circ} \text {, Contraste Haralick } 90^{\circ} \text {, Entropia, Valor } \\
\text { mínimo, Desvio padrão, Variância, Correlação Haralick } 90^{\circ} \text {, Correlação Haralick } 0^{\circ} \text {, Valor máximo, Moda, Desvio } \\
\text { padrão Haralick } 0^{\circ} \text {, Desvio padrão Haralick } 90^{\circ} \text {, Média Haralick } 0^{\circ} \text {, Soma, Média, Média Haralick } 90^{\circ} \text {, Energia } \\
\text { Haralick } 90^{\circ} \text {, Energia Haralick } 0^{\circ} \text {, Registrabilidade (rotação), QuiSquare Haralick } 90^{\circ} \text {, Registrabilidade } \\
\text { (translação), QuiSquare Haralick } 0^{\circ}\end{array}$ \\
\hline 0,2 & $\begin{array}{l}\text { Dissimilaridade Haralick } 0^{\circ} \text {, Contraste Haralick } 0^{\circ} \text {, Entropia Haralick } 0^{\circ} \text {, Dissimilaridade Haralick } 90^{\circ} \text {, } \\
\text { Homogeneidade Haralick } 0^{\circ} \text {, Entropia Haralick } 90^{\circ} \text {, Homogeneidade Haralick } 90^{\circ} \text {, Contraste Haralick } 90^{\circ} \text {, } \\
\text { Entropia, Desvio padrão }\end{array}$ \\
\hline 0,3 & $\begin{array}{l}\text { Dissimilaridade Haralick } 0^{\circ} \text {, Entropia Haralick } 0^{\circ} \text {, Entropia Haralick } 90^{\circ} \text {, Dissimilaridade Haralick } 90^{\circ} \text {, } \\
\text { Homogeneidade Haralick } 0^{\circ} \text {, Homogeneidade Haralick } 90^{\circ}\end{array}$ \\
\hline 0,4 & $\begin{array}{l}\text { Dissimilaridade Haralick } 0^{\circ} \text {, Entropia Haralick } 0^{\circ} \text {, Entropia Haralick } 90^{\circ} \text {, Contraste Haralick } 0^{\circ} \text {, Dissimilaridade } \\
\text { Haralick } 90^{\circ} \text {, Homogeneidade Haralick } 90^{\circ} \text {, Homogeneidade Haralick } 0^{\circ}\end{array}$ \\
\hline 0,5 & $\begin{array}{l}\text { Dissimilaridade Haralick } 0^{\circ} \text {, Contraste Haralick } 0^{\circ} \text {, Entropia Haralick } 0^{\circ} \text {, Entropia Haralick } 90^{\circ} \text {, Dissimilaridade } \\
\text { Haralick } 90^{\circ} \text {, Entropia, Homogeneidade Haralick } 0^{\circ}\end{array}$ \\
\hline 0,6 & $\begin{array}{l}\text { Dissimilaridade Haralick } 0^{\circ} \text {, Contraste Haralick } 0^{\circ} \text {, Dissimilaridade Haralick } 90^{\circ} \text {, Entropia Haralick } 0^{\circ} \text {, Entropia } \\
\text { Haralick } 90^{\circ} \text {, Homogeneidade Haralick } 0^{\circ}\end{array}$ \\
\hline 0,7 & Contraste Haralick $90^{\circ}$, Entropia Haralick $90^{\circ}$, Entropia, Dissimilaridade Haralick $90^{\circ}$, Contraste Haralick $0^{\circ}$ \\
\hline 0,8 & $\begin{array}{l}\text { Homogeneidade Haralick } 0^{\circ} \text {, Desvio padrão, Variância, Homogeneidade Haralick } 90^{\circ} \text {, Correlação Haralick } 0^{\circ} \text {, } \\
\text { Correlação Haralick } 90^{\circ} \text {, Registrabilidade (translação) }\end{array}$ \\
\hline 0,9 & QuiSquare Haralick $0^{\circ}$, Valor máximo, Variância, Desvio padrão Haralick $90^{\circ}$, Média Haralick $90^{\circ}$, Soma \\
\hline
\end{tabular}

\section{Desempenho dos modelos de classificação}

Para avaliar o comportamento de diferentes classificadores usando os conjuntos de amostras gerados pela etapa anterior, foi utilizado o sistema WEKA 3.6.3 (Holmes, Donkin, and Witten 1994) que é um pacote de software gratuito que contém recursos para análise de dados e diferentes técnicas para aprendizado computacional. Este pacote permite efetuar análise exploratória de dados e atributos como também permite testar e avaliar o desempenho de vários algoritmos clássicos da literatura. Também é possível exportar instâncias de modelos de classificadores treinados internamente. Estas instâncias podem ser usadas a posteriori para classificar (predizer) outras amostras que não foram usadas no treinamento.

Para cada conjunto de amostras foram calculados os parâmetros dos quatro modelos de classificadores, escolhidos de forma a representar quatro classes distintas: Naive Bayes, classificador probabilístico simples, baseado no teorema de Bayes (John and Langley 1995). IBk (Aha, Kibler, and Albert 1991), que é uma extensão do modelo de vizinho mais próximo (Cover and Hart 1967). AdaBoostM1 (Freund and Schapire 1996) que é uma das variações do algoritmo de Boosting e como tal os conjuntos de treinamento são montados iterativamente a partir do conjunto inicial. J48, que é implementação do algoritmo C4.5 (Quinlan 1993) baseado em árvores de decisão.

Os atributos utilizados são aqueles que foram selecionados para cada conjunto de amostras. Em todos os casos, para validação, foi usado o conjunto de todas as amostras, ou seja, foram validadas inclusive as amostras que não foram aleatoriamente selecionadas para fazer parte do conjunto de treinamento, totalizando 878 amostras de validação. 
Os modelos e parâmetros utilizados para a execução dos experimentos foram: Naive Bayes com uso de um estimador baseado em kernel; sem o uso de discretização supervisionada. IBk com parâmetro de 3 vizinhos mais próximos; sem validação cruzada; sem pesos por distância; sem média quadrada; tamanho de janela 0; busca linear por distância euclidiana; com normalização de distâncias e sem inversão de seleção. AdaBoostM1 - com parâmetro de 10 iterações; 1 como valor da semente; sem uso de reamostragem; 100 como valor de limiar de distância; NaiveBayes como classificador fraco; com estimador baseado em kernels; sem discretização. J48 - sem partições binárias; fator de confidência 0,25 ; número mínimo de objetos 2; 3 partições; sem balanceamento por redução de erro; 1 como valor de semente; com crescimento de sub-árvore; com balanceamento e sem uso de Laplace.

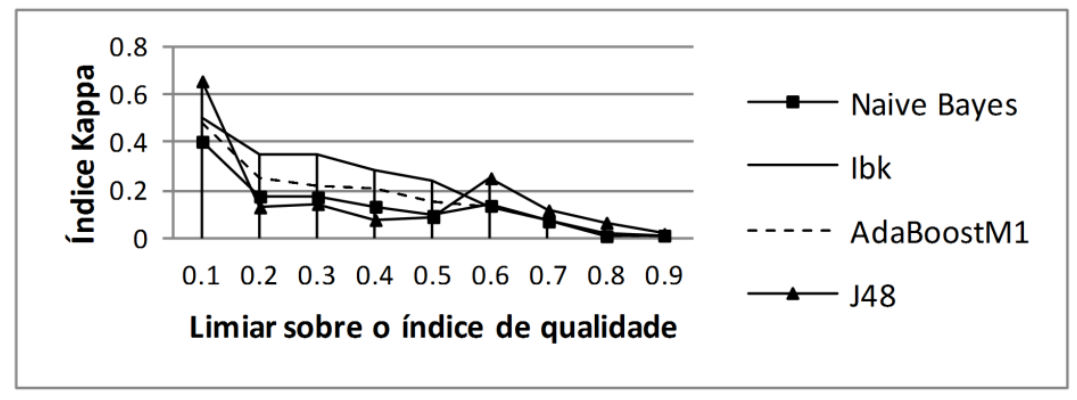

Figura 2 : Resultado da avaliação dos classificadores

O resultado global é apresentado na Erro! Fonte de referência não encontrada., em que o desempenho dos classificadores é medido segundo o índice Kappa (Siegel and Castellan 1988), que é uma medida de concordância, normalizado entre zero e um, onde o valor um representa o máximo de concordância do resultado em relação às amostras. Maiores valores de índice Kappa indicam que um modelo de classificação possui melhor capacidade de predizer corretamente as classes. Nesta figura é possível verificar que para os casos intermediários, onde o valor do limiar de qualidade está em torno de 0,5 , o classificador Ikb se destaca com melhores valores de índice Kappa. Para os casos extremos, onde o valor do limiar de qualidade é 0,1 ou acima de 0,6 (neste caso onde o número de amostras é baixo) o classificador J48 apresentou melhor desempenho.

\section{Desempenho computacional obtido na correção geométrica}

Para avaliar qual é o desempenho computacional obtido pela aplicação da técnica de classificação foram feitos dois experimentos onde o processo de correção das imagens foi refeito usando somente os kernels rotulados como de boa qualidade por um classificador. O primeiro experimento foi feito com kernels classificados por um classificador Ibk, treinado com amostras do grupo onde o limiar de índice qualidade mínimo é 0,5 (dentre os nove grupos de amostras citados anteriormente gerados pela variação do índíce mínimo de qualidade do kernel). $\mathrm{O}$ segundo experimento foi feito com kernels classificados pelo modelo de um classificador J48, treinado com amostras do grupo onde o limiar de índice qualidade mínimo é 0,1 . Para que seja possível a comparação com a situação onde a correção é feita usando todos os kernels (sem classificação), foram usados os mesmos parâmetros de correção geométrica das imagens nesta 
situação. Os modelos dos classificadores usados para os experimentos foram selecionados por apresentarem melhor desempenho segundo os índices mínimos de qualidade escolhidos.

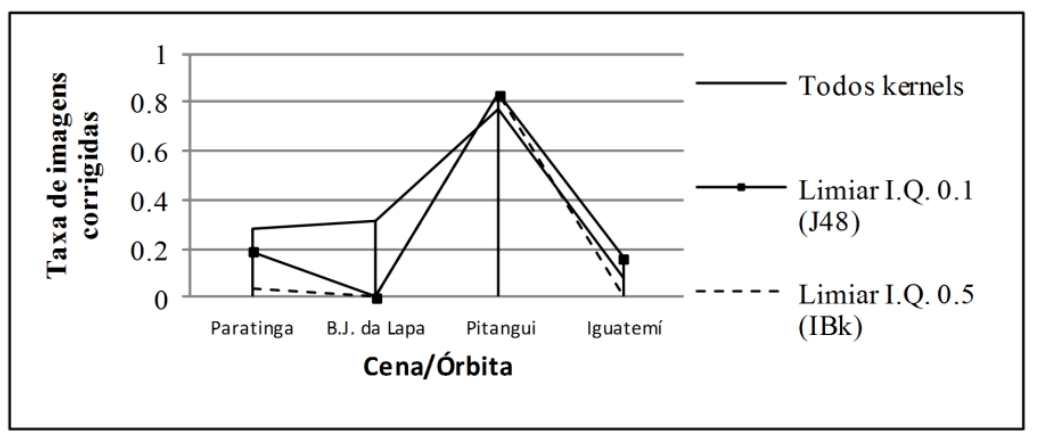

Figura 3: Comparação de taxa de imagens corrigidas

Para a classificação dos kernels foi utilizada a funcionalidade provida pelo software WEKA, que permite a exportação do modelo de classificação e seu uso a posteriori para predizer a classe de novas amostras. O resultado comparativo da taxa de imagens corrigidas por cena (razão entre o número de cenas efetivamente corrigidas e o número total de cenas submetidas ao processo de correção) é mostrado na Erro! Fonte de referência não encontrada.. Verifica-se que a taxa de imagens corrigidas tem um decréscimo para aquelas cenas com baixa quantidade de kernels disponíveis para a correção, como é o caso das cenas de Paratinga (em média 160,38 kernels), Bom Jesus da Lapa (em média 103,08 kernels) e Iguatemí (em média 103,54). Tal fato aponta que alguns dos kernels, que seriam usados com sucesso na correção geométrica, foram incorretamente classificados como kernels ruins e foram descartados. Em contrapartida para a cena de Pitanguí (em média 228,16 kernels), onde existe maior disponibilidade de kernels, houve melhora na taxa de imagens corrigidas.

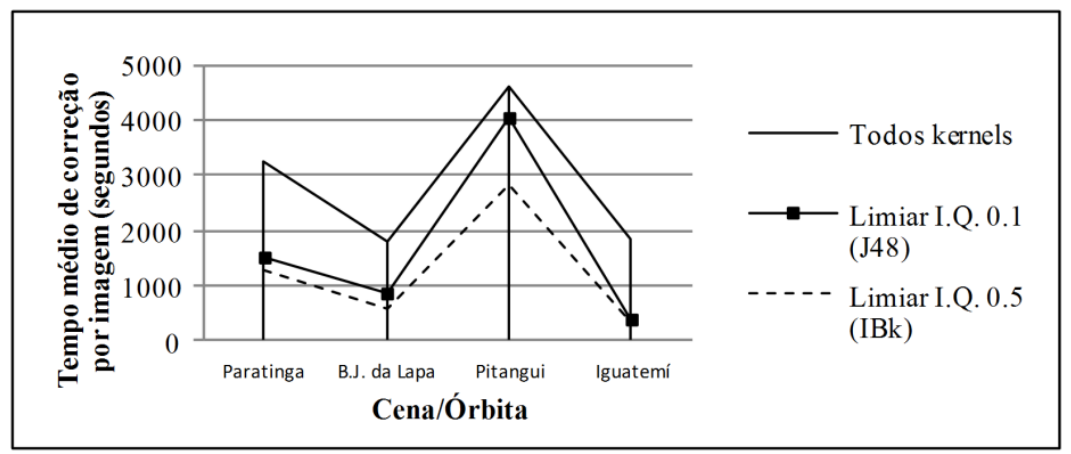

Figura 4: Comparação do tempo de execução

A Figura 4 mostra a comparação entre o tempo médio gasto na correção de cada imagem para cada um dos experimentos. Nesta figura verifica-se que mesmo usando um modelo de classificador relativamente simples, como o IBk, é possível executar o processo de correção em aproximadamente metade do tempo. Para o caso do classificador J48, em que o desempenho do classificador é maior (em relação ao índice Kappa) ainda existe ganho expressivo de velocidade.

A Figura 5 mostra a razão entre o número médio de kernels que efetivamente definiram os parâmetros da transformação geométrica (kernels usados com sucesso) e o número médio de kernels processados (correlacionados e não-correlacionados). Verifica-se que em todas as situações a taxa de kernels úteis é maior, quando comparando com o experimento onde todos os kernels são usados. 


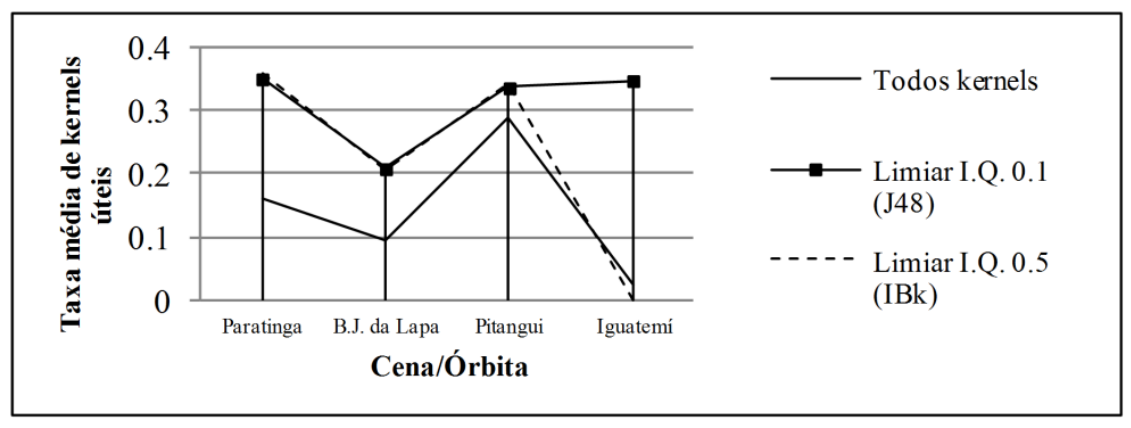

Figura 5: Comparação da taxa de kernels úteis

\section{Conclusão}

Neste trabalho foi proposta uma metodologia onde o principal objetivo é a redução do tempo de computação no processo de correção geométrica automática de imagens orbitais. Para tal é aplicada uma técnica de seleção automática de kernels, utilizados no processo de correção, que se baseia no uso de um classificador treinado utilizando amostras cujos atributos são extraídos da informação radiométrica de cada kernel.

Pelos resultados obtidos, verificou-se que o classificador J48 trouxe melhores resultados, em termos de tempo de execução e número de cenas corrigidas. Nesta situação ele foi capaz de selecionar aqueles kernels que darão origem a pontos de controle que serão efetivamente usados para o cálculo da transformação geométrica.

Considerando os resultados obtidos para todas as regiões escolhidas foi possível obter um ganho de desempenho expressivo: Para o caso do uso dos kernels classificados pelo classificador J48, o tempo médio de execução é $50 \%$ inferior ao tempo de correção usando todos os kernels. E a taxa de kernels efetivamente usados na correção é praticamente duplicada em alguns casos.

Foi mostrado que o objetivo proposto neste trabalho, que é a melhoria do processo de correção, foi alcançado. Para tal foram utilizados modelos relativamente simples de classificadores, tomando como referência um limiar baixo de índice de qualidade. Desta forma foi possível obter um aumento expressivo no desempenho pela redução no tempo gasto de processamento. Isto se deve principalmente ao fato de que a grande maioria dos kernels tem baixo índice de qualidade.

O tempo gasto em processos relativos à seleção de kernels (criação de amostras de classificação, treinamento de um classificador e classificação de kernels) é expressivamente menor que o tempo gasto na correção das imagens em si. Desta forma é válido o estudo de novos atributos relativos aos kernels: período de aquisição, condições de aquisição, tipo de sensor além de novos atributos radiométricos mais complexos. Da mesma forma é válido o estudo de modelos mais complexos de classificadores, principalmente aqueles que tenham maior robustez para tratar a situação onde o número de amostras é baixo. 


\section{REFERÊNCIAS BIBLIOGRÁFICAS}

Aha, D. W.; Kibler, D.; Albert, M. K. Instance-based learning algorithms. Journal of Machine Learning, v.6, n.1, p.37-66, 1991.

Câmara, G., et al. TerraLib: Technology in Support of GIS Innovation. in II Workshop Brasileiro de Geoinformática, GeoInfo2000. 2000. São Paulo.

Castejon, E. F. et al. Avaliação de métodos de casamento de imagens para mosaico de imagens orbitais. In: Simpósio Brasileiro De Sensoriamento Remoto, 14., 2009, Natal. Anais... São Jose dos Campos: INPE, 2009. p. 6805-6812.

Castejon, E. F. Classificação de amostras de imagens geo-referenciadas para correção geométrica de imagens dos satélites CBERS. 2011. 118 folhas. Tese de mestrado em Engenharia Eletrônica e Computação/Informática - Instituto Tecnológico de Aeronáutica, São José dos Campos.

Castejon, E. F.; Fonseca, L. M. G.; Arcanjo, J. S. Melhoria da geometria e posicionamento de imagens orbitais de média resolução - Um experimento com dados CBERS-CCD. In: Simpósio Brasileiro De Sensoriamento Remoto, 16. (SBSR), 2013, Foz do Iguaçu. Anais... São José dos Campos: INPE, 2013. p. 8048-8055. DVD, Internet. ISBN 978-85-17-00066-9 (Internet), 97885-17-00065-2 (DVD). Disponível em: <http://urlib.net/3ERPFQRTRW34M/3E7GJU8>. Acesso em: 19 set. 2014.

Chalermwat, P. High performance automatic image registration for remote sensing. 1999. Tese (Doutorado) - George Mason University, Fairfax.

Cover, T. M.; Hart, P. E. Nearest neighbor pattern classification. IEEE Transactions on Information Theory, v.13, 21-27. 1967.

D' Alge, J. C. L.; Souza, R. C.; Erthal, G. J. Geometric quality assessment of CBERS-2 images. In: Simposio Latinoamericano Sobre Percepcion Remota Y Sistemas De Informacion Espacial, 2004, Santiago. Anales... São Jose dos Campos: INPE, 2004, 2004. INPE-13309-PRE/8551.

Deng, W. et al. Off-Line chinese writer identification based on character-level decision combination. In: International Symposiums On Information Processing, 2008. Proceedings... Cambridge: IEEE, 2008. p.762-765 ISBN: 978-0-7695-3151-9.

Eikvil, L.; Holden, M.; Huseby, R. B. Adaptive registration of remote sensing images using supervised learning. Photogrammetric Engineering \& Remote Sensing, v.75, n.11, p.1299-1306, 2009.

Fedorov, D. Sistema Semi-Automático de Registro e Mosaico de Imagens. 2002. 150f. Dissertação (Mestrado em Computação Aplicada) - INPE, São José dos Campos.

Fischler, M.A.; Bolles, R.C. Random sample consensus: a paradigm for model fitting with applications to image analysis and automated cartography. In: Readings in computer vision: issues, problems, principles, and paradigms. San Francisco: Morgan Kaufmann, 1987.

Fisher, R. A. The use of multiple measurements in taxonomic problems. Annals Of Human Genetics. v. 7 n.2, p. 179-188, 1936. DOI: 10.1111/j.1469-1809.1936.tb02137.x.

Fonseca, L.M.G. Registro Automático de Imagens de Sensoriamento Remoto baseado em Múltiplas Resoluções. 1999. Tese (Doutorado em Computação Aplicada) - Instituto Nacional de Pesquisas Espaciais, São José dos Campos.

Freund, Y; Schapire, R. E. Experiments with a New Boosting Algorithm. In: Machine Learning International Conference, 13., 1996. Proceedings... [S.l.: s.n.], 1996. p.148-156. 
Gugan, D.J., Dowman, I.J., 1988. Accuracy and completeness of topographic mapping from SPOT imagery. Photogrammetric Record. 12 (72), 787-796.

Haralick, R.M. Statistical and Structural Approaches to Texture. Proceedings of the IEEE, v.67, p.786-804, 1979.

Herold, M.; Liu, X.; Clarke, K. Spatial metrics and image texture for mapping urban land use. Photogrammetric Enggineering \& Remote Sensing, v.69, p.991-1001, 2003.

Holmes, G.; Donkin, A.; Witten, I.H. Weka: a machine learning workbench. In: Australia And New Zealand Conference On Intelligent Information Systems, 2., 1994, Brisbane. Proceedings... Piscataway: IEEE, 1994. p.357-361. DOI 10.1109/ANZIIS.1994.396988.

John, H.; Langley, P. Estimating continuous distributions in bayesian classifiers. In: Conference On Uncertainty In Artificial Intelligence, 11., 1995, San Mateo. Proceedings... Montreal: Morgan Kaufmann, 1995. p.338-345.

Kim, T., Dowman, I., 2006. Comparison of two physical sensor models for satellite images: position-rotation model and orbit-attitude model. The Photogrammetric Record. 21 (114), 110123.

Marcato Junior, J.; Tommaselli, A. M. G., 2013. Exterior orientation of CBERS-2B imagery using multi-feature control and orbital data. ISPRS Journal of Photogrammetry and Remote Sensing, 79 (2013), 219-225.

Moravec, H. P. Towards automatic visual obstacle avoidance. In: Intenational Joint Conference On Artifical Intelligence, 5., 1977, Cambridge, MIT. Proceedings... Cambridge: MIT, 2005. p. 584.

Pratt, W. K. Correlation techniques of image registration. IEEE transactions on aerospace and eletronic systems, v.10, n.3, p.353-358, 1974.

Queiroz, C. J. Análise de transformações geométricas para o georreferenciamento de imagens do satélite CBERS-1. 2002. Tese de mestrado em Sensoriamento Remoto - Universidade Federal do Rio Grande do Sul, Porto Alegre - RS.

Quinlan, J. R. C4.5: programs for machine learning. San Mateo: Morgan Kaufmann Publishers, 1993.

Sidney, S.; Castellan, N. J. Jr. Nonparametric statistics for the behavioral sciences. 2nd Ed. New York: McGraw-Hill, 1988.

Silva, A. J. F. M. Geometria de imagens: do projeto do satélite à geração dos produtos. 2007. 256f. Tese (Doutorado em Sensoriamento Remoto) - INPE, São José dos Campos.

USGS. Extraction of GCP chips from GeoCover using Modified Moravec Interest Operator (MMIO) algorithm. The United States Geological Survey (USGS) Online Repository, 2006. Disponível em:

<http://landsat.usgs.gov/documents/MMIO_GeoCover_Control_White_Paper.pdf >. Acesso em: 14 de Julho de 2011.

Yu L.; Liu H. Efficient feature selection via analysis of relevance and redundancy. Journal of Machine Learning Research, v.5, p.1205-1224, 2004.

Zitová, B.; Flusser, J. Image registration methods: a survey. In: Image and Vision Computing, 21, p. 977-1000, 2003.

Recebido em Maio de 2014.

Aceito em Agosto de 2015. 